\title{
DESEQUILIBRIOS BANCARIOS Y RIESGO SISTÉMICO: UNA APROXIMACIÓN CON TEORÍA DE LOS JUEGOS
}

\section{BANK IMBALANCES AND SYSTEMIC RISK: AN APPROACH WITH THE \\ GAME THEORY}

Demian Macedo*1, Matias Cabrera ${ }^{2}$, Jorge Mauricio Oviedo ${ }^{3}$

\footnotetext{
1 Departamento de Economía de la Empresa. Universidad de las Islas Baleares, España. E-mail: demian.macedo@uib.es 2 BBVA Research, España. E-mail: matiasdaniel.cabrera@bbva.com

3 Instituto de Economía y Finanzas y Departamento de Estadística y Matemática. Facultad de Ciencias Económicas. Universidad Nacional de Córdoba.

E-mail: joviedo@eco.unc.edu.ar
}

RESUMEN

En este trabajo se buscará analizar, desde la perspectiva de teoría de los juegos, las interrelaciones que se generan entre los agentes del sistema financiero ante una situación de riesgo sistémico generado por un banco que se encuentra atravesando un proceso de insolvencia. Para ello, el modelo que se propone cuenta con tres actores: un banco en dificultades que pasa por un proceso de quiebra, un banco de mayor tamaño que tiene la posibilidad de adquirirlo, y un Banco Central que debe decidir si interviene en el mercado para rescatar al banco en quiebra (y el momento óptimo para hacerlo). En un contexto en el que el potencial comprador tiene incentivos para demorar su acción, con el objetivo de reducir el precio de compra, el modelo pone de manifiesto la necesidad de que el Banco Central cuente con suficiente poder de persuasión y pueda ejecutar una amenaza creíble como estrategia para lograr la estabilidad financiera.

Palabras clave: Riesgo Sistémico, Pánicos Bancarios, Equilibrio Perfecto en Subjuegos

\section{ABSTRACT}

This paper seeks to analyze, from a game theory perspective, the interrelationships between agents in the financial system. Particularly, we focus on a situation in which agents face systemic risk arising from a bank that is going through an insolvency process. With this objective, the proposed model presents three agents: a bank going through financial difficulties, a larger bank which has the option of taking-over the troubled bank, and a Central Bank that must decide whether to intervene in the market to bailout the troubled bank (and the precise moment for its intervention). If the potential buyer has incentives to delay its decision to buy the troubled bank (in order to reduce the purchase price), the model shows that the Central Bank needs to be sufficiently persuasive and should be able to execute a credible threat as a strategy if it seeks to preserve financial stability.

Keywords: Systemic risk, banking panics, Subgame perfect equilibrium 


\section{INTRODUCCIÓN}

Comúnmente se denomina riesgo sistémico a situaciones en las que la estabilidad del sistema financiero en su conjunto se encuentra en peligro. Este riesgo, creado por las interdependencias que existen el sistema, puede llevar a que la inestabilidad en una institución se contagie al resto del sistema generando un efecto dominó. Esta inestabilidad se puede manifestar de diversas formas: desde un congelamiento de la liquidez en los mercados interbancarios (Acharya \& Skeie, 2011), hasta un pánico bancario que lleve a un retiro generalizado de los depósitos (Diamond \& Dybvig, 1983; Saunders y Wilson, 1996; Goldstein \& Pauzner, 2005). Estos problemas pueden tener orígenes diversos: problemas del soberano (Alter \& Schüler, 2012; Acharya, Drechsler, \& Schnabl, 2014; Cabrera, Dwyer \& Samartín-Saénz, 2016), burbujas financieras que lleven a un proceso de insolvencia generalizada (Brunnermeier \& Oehmke, 2013), marcos de regulación financiera inapropiados (Levine, 2012) o problemas de información asimétrica y riesgo moral (Faria-e-Castro, 2016).

En la actualidad, el riesgo sistémico, los pánicos y las corridas bancarias constituyen una de las mayores preocupaciones de los bancos centrales, tanto en países en vías de desarrollo como en el mundo desarrollado (Bullard, Neely \& Wheelock, 2009). En ese sentido, adoptar y diseñar medidas y reglas de comportamiento tendientes a velar por la salud y estabilidad del sistema financiero ha sido uno de los principales deberes de los bancos centrales y los reguladores financieros, particularmente desde la última crisis (Fernández, 1996; Acharya, 2015; Fischer, 2016; Cabrera, Dwyer \& Nieto, 2018).

Pero para comprender los canales de contagio que generan riesgo sistémico es necesario entender las interrelaciones entre los agentes financieros. Existen muchos intentos de formalizar las relaciones y factores que llevan a una crisis sistémica (Freixas \& Parigi, 1998; Allen \& Gale, 2000; Rochet \& Vives, 2004; Vives, 2014). Algunos trabajos buscan definir estas relaciones en base a la teoría de redes (Paltalidis, Gounopoulos, Kizys \& Koutelidakis, 2015; Acemoglu, Ozdaglar \& Tahbaz-Salehi, 2015). Otros enfoques más tradicionales emplean modelos de "global games" (Goldstein \& Pauzner, 2005) o de equilibrio general. En este último caso, los modelos introducen shocks de consumo a los depositantes (Freeman, 1996) que llevan a un desequilibrio en el sector bancario, el cual es susceptible de contagiarse al resto del sistema, llevando a un proceso de insolvencia generalizada.

Una vez que estas relaciones son analizadas, se plantea la posibilidad de un rescate público al sector financiero, por ejemplo mediante la intervención del banco central. En este caso, las autoridades pueden prestar ayuda a las instituciones con desequilibrios, o dejarlas quebrar, dependiendo de diferentes factores que pueden entrar en juego. Aquí se supone que las autoridades (en este caso el banco central) buscan minimizar el riesgo sistémico. Sin embargo, en la mayoría de los casos, no se contempla que existe una otra alternativa que la intervención pública, dejando sin consideración una opción que puede ser superadora si se realizara de forma eficiente: dejar que otro banco adquiera a la institución en problemas a precios de liquidación ${ }^{1}$.

El objetivo de este trabajo es precisamente estudiar estas situaciones en las que un banco en dificultades puede ser comprado por otro de mayor capacidad, analizando cómo el riesgo sistémico puede llegar a ser exacerbado si los bancos tienen comportamientos estratégicos. En particular, nos enfocamos en una situación en la que el banco solvente decide demorar la compra del banco en dificultades, con el 
objeto de reducir el precio de venta en la mayor cuantía posible. Si bien este comportamiento es óptimo desde el punto de vista de los agentes, dados los incentivos que tiene, puede traer aparejado un incremento del riesgo sistémico.

Para ello, utilizando teoría de los juegos, modelizamos y analizamos las interrelaciones que se generan entre los agentes del sistema financiero. Para ello, supondremos que existen tres agentes que participan en esta economía: el banco que atraviesa situación de quiebra, un banco de mayor tamaño con posibilidades de adquirirlo, y un Banco Central que tiene el poder de rescatar al banco en dificultades (o dejarlo caer en su defecto). El modelo permitirá analizar las interrelaciones entre los agentes y derivar situaciones de equilibrio para promover la estabilidad financiera.

El trabajo se estructura de la siguiente manera. En la Sección I se describe el problema de los agentes. En la Sección II se formaliza el modelo, y se presentan soluciones alternativas al modelo. En la Sección III se presentan posibles extensiones al modelo. Finalmente, se presentan las conclusiones.

\section{METODOLOGÍA}

Utilizando un enfoque basado en la teoría de los juegos, definiremos un modelo algebraico que nos permite derivar de forma algebraica las decisiones que los agentes tomarán. Pero antes de definir dichas reglas de decisión, es necesario describir la problemática de los agentes en el modelo.

El modelo se centra en la posibilidad de quiebra de un banco como causante del riesgo sistémico. Este banco cuenta con la peculiaridad de ser un banco de tamaño mediano que no se ajusta a las recomendaciones de política "too big to fail" (Rochet
\& Tirole, 1996): no es lo suficientemente grande como para garantizar su rescate en todos los escenarios, ni tampoco lo suficientemente pequeño como para que su quiebra sea inocua al resto del sistema.

Un supuesto importante es que, en virtud a la situación que atraviesa, los problemas del banco se acrecientan con el tiempo, con lo cual también se reduce su valor de mercado. Esto podría ilustrarse de la siguiente manera. Un banco está atravesando problemas de liquidez transitorios, pero es solvente, con lo que el valor de su cartera de activos no ha cambiado. Si el mercado de liquidez se cierra para este banco (por cuestiones ajenas a su solvencia), el banco deberá vender activos a precios de liquidación para cubrir sus necesidades de liquidez. Lo que suponemos en este modelo, es que mientras más tiempo transcurre, el precio de liquidación por esa cartera de activos es cada vez menor.

Además hay otros dos actores que pueden tomar partida de la situación. Por un lado, existe otro banco de mayor tamaño-al que se hará referencia como BG (Banco Grande)- que puede estar interesado en la adquisición del banco en problemas, deseando obtener la máxima rentabilidad en esta operación. Para ello intentara realizar la compra al menor precio posible, el cual se supuso es decreciente con el tiempo ${ }^{2}$.

Por otro lado, se encuentra el Banco Central - al que se hará referencia como BCquien debe controlar la salud del sistema financiero. Asumimos que si el BC interviene, es capaz de implementar un plan de regularización y saneamiento, o puede actuar como prestamista de última instancia. Además, se asume que una intervención es siempre costosa en términos de credibilidad. Así, en cuanto mayor y más temprano sea el salvataje, mayor es la pérdida de credibilidad frente a otras instituciones, exacerbando el problema del ries-

\footnotetext{
2 Para simplificar el problema, se asumirá que el banco no tiene en cuenta otras cuestiones como por ejemplo el efecto que la compra tendrá en su propia
} cartera de activos, u otras cuestiones estratégicas como posibles ganancias de eficiencia por duplicación de costes respecto a la entidad que adquiere. 
go moral y como consecuencia del riesgo sistémico.

A su vez, asumimos que, si el banco en peligro no es rescatado o adquirido por otro banco antes de su quiebra, existe la posibilidad de contagio al resto del sistema. Por lo tanto, el BC ha de considerar cuidadosamente las distintas posibilidades $^{3}$. Esto significa que estará dispuesto a extender la situación sólo hasta un determinado momento (antes de rescatarlo o de que el BG decida comprar a la entidad en problemas).

Así queda de manifiesto que existen dos entidades participantes en este juego, BC y BG, cuyas estrategias óptimas de comportamiento estarán influenciadas por la decisión del otro agente.

\section{A - PROBLEMA DEL BC:}

En su intento de preservar la salud del sistema financiero, el BC se enfrenta al problema de minimizar una función de riesgo sistémico (RS) que depende de dos argumentos. Por un lado, dicha función depende del momento " $t$ " en que el BC hace efectiva su decisión de rescatar a la unidad financiera para limitar el pánico y evitar así una posible corrida bancaria. Dicha variable es denominada $\mathrm{t}^{\mathrm{BC}}$. Por otro lado, el RS también depende de los posibles "momentos" en que el banco interesado (BG) decide hacer efectiva su decisión de adquirir el banco con problemas. Esta variable se la designa con $\mathrm{t}^{\mathrm{G}}$.

Con respecto a la variable $\mathrm{t}^{\mathrm{BC}}$ la función de RS debe tener en cuenta dos efectos: por un lado, debe reflejar el incremento del RS por rescatar anticipadamente al banco en peligro lo que incrementa el riesgo moral (la cual denominaremos $\mathrm{RM}\left(\mathrm{t}^{\mathrm{BC}}\right)$ ), y por otro el incremento de RS por rescatarlo tardíamente en donde la difusión del pánico puede ser ya irremediable (denominado $\left.\mathrm{RC}\left(\mathrm{t}^{\mathrm{BC}}\right)\right)$. De ésta forma, representaremos la función de riesgo sistémico como una suma ponderada de esos riesgos. Suponemos además que dicha función es dos veces diferenciable y que alcanza un mínimo en $\mathrm{t}^{\mathrm{BC}}=\mathrm{a}$. Es decir, en este momento los efectos marginales del tiempo sobre $\mathrm{RC}($.$) y \mathrm{RM}($.$) son iguales.$

En segundo lugar, definimos $t^{*}$ como el valor máximo de espera del $\mathrm{BC}$, a que el BG adquiera al banco en peligro. Si dicha adquisición se produce antes de $\mathrm{t}^{*}$, asumimos que el RC se hace nulo, por lo que el valor del RS será únicamente debido a la componente de RM. Esto es importante pues nos da los lineamientos para determinar $t^{*}$, siendo este el valor que iguala $\mathrm{RS}\left(\mathrm{t}^{*}\right)$ con el valor minimizado de la función RS como suma ponderada de ambos riesgos. Es decir se debe resolver:

$$
\mathrm{RM}\left(\mathrm{t}^{*}\right)=\alpha \mathrm{RM}(\mathrm{a})+(1-\alpha) \mathrm{RC} \text {. }
$$

Si BG decide adquirir al banco en problemas antes del momento crítico t*, entonces RS experimentará una derivada continuamente negativa en todo su dominio con respecto a $\mathrm{t}^{\mathrm{BC}}$ reflejando que la decisión óptima en estas circunstancias será no rescatar al banco en problemas.

A su vez, existe un momento "C" tal que si el banco en problemas no fue rescatado, o no ha sido adquirido por BG, el sistema financiero colapsa $\left(\mathrm{t}^{* *}\right)$. Algebraicamente los supuestos antes mencionados pueden traducirse en la siguiente expresión:

$$
R S\left(t^{G}, t^{B C}\right)=\left\{\begin{array}{lr}
\alpha R M\left(t^{B C}\right) & \forall t^{B C} \wedge \forall t^{G}<t^{*} \\
\alpha R M\left(t^{B C}\right)+(1-\alpha) R C\left(t^{B C}\right) & \forall t^{B C}<t^{* *} \wedge \forall t^{G}>t^{*} \\
\infty & \forall t^{B C}>t^{* *} \wedge \forall t^{G}>t^{*}
\end{array}\right.
$$

donde RM y RC satisfacen las siguientes condiciones de regularidad:

\footnotetext{
${ }^{3}$ En el análisis, nos abstraemos de otras cuestiones que podrían ser relevantes a la hora de tomar esta decisión, como por ejemplo, el costo económico del rescate en sí mismo.
} 
$\mathrm{RM}^{\prime}<\mathrm{o} ; \mathrm{RC}^{\prime}>\mathrm{o} ; \mathrm{RC}^{\prime}>\mathrm{R} M^{\prime}$.

Como se aprecia $\mathrm{RS}\left(\mathrm{t}^{\mathrm{G}}, \mathrm{t}^{\mathrm{BC}}\right)$ es una función de dos variables y cuya expresión puede descomponerse en tres tramos: primero cuando el BG adquiere antes de $\mathrm{t}^{*}$, segundo cuando el BG adquiere después de $t^{*}$, y tercero cuando el BG adquiere después de $\mathrm{t}^{*}$ y $\mathrm{BC}$ decida rescatarlo después de $t^{* *}$.

Como se dijo el problema del BC es:

$$
\min _{t^{B C}} R S=R S\left(t^{G}, t^{B C}\right)
$$

cuya solución óptima es:

$$
t_{\text {opt }}^{B C}=f\left(t^{G}\right)= \begin{cases}\infty & ; \forall t^{G}<t^{*} \\ a ; a \in\left(0, t^{*}\right) ; & ; \text { si } t^{G}>t^{*}\end{cases}
$$

Lo que no es más que decir que si $\mathrm{t} \mathrm{C}<$ $\mathrm{t}^{*}$, el $\mathrm{BC}$ no intentará jamás rescatar al banco en peligro, pues la adquisición por parte del BG se produjo a tiempo. De lo contrario su estrategia óptima es rescatarlo en el momento en que se minimizan la suma de los efectos considerados con anterioridad. Como se aprecia, la decisión óptima del BC está condicionada por la decisión que tome el BG. Esta condicionalidad servirá posteriormente para justificar que dicha solución óptima constituye una función de reacción ante las decisiones posibles del BG.

\section{$B$ - EL PROBLEMA DEL BG:}

Al evaluar la posibilidad de adquisición, éste intentará maximizar los beneficios esperados de tal acción. Su función de beneficios está compuesta por dos elementos: Los ingresos futuros y el precio al cual adquiere al banco en problemas de bancarrota. En cuanto a los beneficios supondremos en son constantes e independientes del tiempo, siempre que la adquisición se haga antes del rescate del BC. En cuanto al precio de adquisición, el BG se comportará de manera estratégica intentando retrasar al máximo su momento de compra (siempre que sea anterior al rescate del $\mathrm{BC})$. Recuerde que se ha asumido que el precio de compra es decreciente en el tiempo. Por otro lado, si el BC rescatase el banco antes de que el BG lo adquiriera, sus beneficios esperados se reducen automáticamente a cero, lo que refleja el hecho de que el BC se perdió el negocio.

Lo mencionado anteriormente se puede representar algebraicamente de la siguiente manera:

$$
\begin{aligned}
& \max _{t^{G}} B T\left(t^{G}, t^{B C}\right)= \begin{cases}\beta I F-P\left(t^{G}\right)>0 ; t^{G}<t^{B C} \\
0 & t^{G}>t^{B C}\end{cases} \\
& P^{\prime}<0
\end{aligned}
$$

Donde " $\beta$ " representa un coeficiente de expectativas subjetivas por parte del BC en relación con los beneficios futuros esperados en caso de adquirir al banco en problemas. En una primera instancia consideraremos el caso en que este coeficiente es determinístico y conocido por todos los demás agentes de la economía incluido el BC. A su vez, "IF" representa el ingreso futuro que espera obtener el BG por la compra. Se supone que este es constante e independiente del tiempo.

La solución al problema de maximización del BG es:

$$
\max _{t^{g}} B T\left(t^{G}, t^{B C}\right) \rightarrow t^{G}=t^{B C}-\varepsilon
$$

Donde " $\varepsilon$ " representa a una cantidad de tiempo positiva en infinitesimalmente pequeña.

Así, dados nuestros supuestos, los beneficios aumentan con el tiempo de adquisición, por lo que la estrategia óptima del BG consiste en adquirir el banco en peligro un instante antes de 
que el BC lo rescate4.

En las siguientes páginas se plantearán diferentes soluciones al problema, donde todas estas características entrarán a jugar un papel preponderante en el concepto de equilibrio encontrado.

\section{RESULTADOS}

Caso uno: Juego dinámico con información perfecta

En este primer caso se supondrá que el juego consiste en dos etapas y que el BC juega primero decidiendo en que momento desea adquirir el banco en problemas y ante esa acción el BC reacciona de forma óptima. Se supone además que existe información perfecta, es decir las características del juego son de dominio público, por lo tanto, el juego es equivalente a uno donde todas estas decisiones se toman en el instante cero. Es decir, las decisiones se prevén por anticipado y una vez tomadas no pueden modificarse. De esta manera, se podrá obtener la función de reacción de los agentes, para luego aplicar el algoritmo de inducción hacia atrás. Aplicando este algoritmo obtenemos un equilibrio de Nash perfecto en sub-juegos, concepto de solución que elimina las amenazas o estrategias no creíbles de los jugadores . Incluso más, esta solución será única si cada jugador no posee más de una acción óptima.

Este contexto probablemente no sea una situación tan atípica si se asume que el BC puede, de alguna manera, conseguir información sobre la operatoria decisional del BC (específicamente del momento $\left.\mathrm{t}^{*}\right)$. De esta manera, el conocimiento de las estrategias del BC por parte BC, le permite a este último obtener un máximo beneficio de esta partida.

Siguiendo a Gibbons (1993), utilizaremos un diagrama de árbol para tener una visión más clara de lo que está pasando:

Figura 1: Diagrama de árbol del modelo

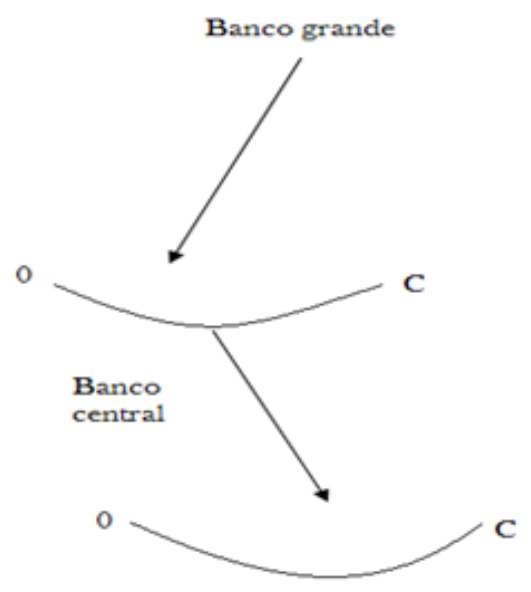

Como se puede observar, tenemos un juego dinámico de dos etapas, en la primera el BG elegirá una acción del espacio continuo de tiempo (representado por la línea ondulada) que varía entre el instante o (cuando comienza a ser factible la compra o rescate del banco en problemas) y el momento $C$ (donde se producirá el caos en la economía). Luego, de la decisión del BC, el BC observa lo ocurrido, y procede a ejecutar su movimiento.

Es muy importante distinguir para la resolución del problema, el momento de inicio de los diferentes sub-juegos. En nuestro caso, al ser el espacio de estrategia del BC continuo, existirán infinitos subjuegos. Estos nacerán en cada punto de la línea curva “o-C". El equilibrio en este tipo de situaciones puede resolverse a través de la función de reacción de cada jugador.

Dado que el BC mueve en última instancia y que la acción óptima de éste depende de la elección de BG, el BG incorpora la función de reacción del BC en su conducta optimizadora. Acorde a la solución por inducción hacia atrás el Equilibrio Perfecto en Sub-juegos se calcula de la siguiente manera:

\footnotetext{
${ }^{4}$ Siempre que se adquiera antes del momento crítico t*. Además, obsérvese como ambas estrategias óptimas dependen de la elección del otro jugador.

5 En este caso particular la amenaza no creíble es que el BC decida rescatar antes del momento t*. Esta acción no es créble, pues de ser así, el BC no está minimizando la función de RS.
} 


$$
\max _{t^{*}} B T\left[t^{G}\left(t^{B C}\right)\right]=\left\{\begin{array}{cc}
\beta I F-P\left(t^{G}\right), & \forall t^{G}<\left\{\begin{array}{cc}
\infty ; \dot{\mathrm{s}} t^{G}<t^{*} \\
a ; \mathrm{s} t^{G}>t^{*}
\end{array}\right. \\
0, & \forall t^{G}> \begin{cases}\infty ; \mathrm{si} t^{G}>t^{*} \\
a ; \mathrm{s} t^{G}>t^{*}\end{cases}
\end{array}\right.
$$

Como puede observarse existen ciertas contradicciones en los subdominios de esta expresión: por ejemplo no puede suceder que " $t G$ " sea menor que "a" ni tampoco que " $t G$ " sea mayor que “ $\infty$ ". Por ello la estructura definitiva queda así:

$$
\max _{t^{G}} B T\left[t^{G}\left(t^{B C}\right)\right]=\left\{\begin{array}{cc}
\beta I F-P\left(t^{G}\right) & \forall t^{G}<t^{*} \\
0 & \forall t^{G}>t *
\end{array}\right.
$$

cuya solución es:

$$
\arg \underset{f^{*}}{\max } B T\left(t^{G}, t^{B C}\right) \rightarrow t^{G} k^{*}=t^{*}-\varepsilon
$$

Así el BG, asumiendo que conoce la forma de operar del $\mathrm{BC}$, puede comportarse estratégicamente y comprar al banco en dificultades en el instante anterior al que el sistema financiero alcance el momento $\mathrm{t}^{*}$, es decir el instante anterior al tiempo máximo que el BC está dispuesto a esperar.

Bajo esta estructura de juego, el BC no puede hacer otra cosa más que dejar que el BG aproveche la oportunidad de comprar el banco en dificultades al menor precio posible. Esto revela la importancia de la información en el juego. Aunque el BC tiene otra herramienta con la que el resultado del juego podría ser diferente.

\section{CASO DOS: BC CON POSIBILIDAD DE REALIZAR AMENAZAS CREÍBLES}

Ahora se concibe la posibilidad de que el BC pueda influir en la percepción que el BG tiene sobre el tiempo máximo $t^{*}$ que el BC estaría dispuesto a esperar. Dicha situación se aproximaría a una situación en la que el BC consigue persuadir al BC y a todo el Sistema Financiero de que intervendrá de forma temprana, pasado cierto límite de tiempo (una amenaza creíble). Dicho poder de persuasión puede transmitirse en un lenguaje más formal porvía de la incorporación deun coeficiente " $\alpha$ " (llamado coeficiente de presión o persuasión) pudiendo este asumir valores solo en el intervalo $[0,1]$. Sustituyendo en las expresiones anteriores $t^{*}$ por " $(1-\alpha) t^{*}$ " el resultado final será:

$$
t_{\text {opt }}^{B G}=(1-\alpha) t^{*}-\varepsilon
$$

Como bien puede apreciarse, a mayor poder de presión o persuasión, el BC puede lograr mejorar el nivel global de RS. Nótese además que el caso 1 no es más que un caso particular en el que $\alpha=0$.

El coeficiente de presión o persuasión ejercida por el $\mathrm{BC}$ puede manifestarse por medio de exigencias de plazo máximo en operaciones varias, o los términos propuestos en una negociación entre los participantes interesados; todas ellas en un contexto que brinde credibilidad hacia las medidas iniciadas.

\section{POSIBLES EXTENSIONES}

Para facilitar la creación de nuevos conocimientos en el presente escrito, el mismo puede expandirse a situaciones más complicadas y mejor interpretativas de la realidad, como, por ejemplo:

a-Considerar situaciones en que los agentes no conocen con exactitud el modo de actuación de los demás, viéndose estos en la necesidad de signar probabilidades a las posibles conductas de los demás agentes. Este escenario podría ser capturado por este modelo vía la incorporación de teoría de juegos con estrategias mixtas.

b- Tener en cuenta la posibilidad de que actúen más agentes en la disputa por querer adquirir el banco en dificultades. A priori, una mayor competencia por la adquisición del banco estimularía la estabilidad financiera. La razón es simple, un incremento en la competencia entre los posibles 
bancos compradores reduciría no solo las intervenciones del Banco Central sino también el grado de especulación de los compradores.

c-Evaluar la posibilidad de que ambas entidades puedan llegar a tener coeficientes de presión, en un contexto dinámico, escenario que se circunscribe en la teoría sobre "Juegos Diferenciales" en analogía a los problemas persecución dinámica.

\section{CONCLUSIONES Y DISCUSIÓN}

En virtud de haber alcanzado el propósito que impulso la consecución del siguiente trabajo, vale destacar que los resultados a los que se arribaron eran un tanto evidentes a raíz de los supuestos en los que se desenvolvía el modelo, sin embargo, su aporte es la formalización de estos.

A través de este modelo hemos probado los patrones de conducta de distintos integrantes del sistema financiero en torno a una de las posibles causas que pueden exacerbar el riesgo sistémico. Se arribó a la conclusión que un BC puede tener poder de influencia sobre conductas estratégicas de bancos interesados en la compra de una institución en problemas. Esto será así en la medida de que el BC sea capaz de llevar adelante acciones creíbles que modifiquen la percepción que los bancos interesados tienen sobre el tiempo máximo de espera para rescatar al banco en problemas.

El marco normativo necesario para afrontar con éxito un problema como el tratado requiere un sistema de incentivos que desalienten las conductas dealto riesgo especialmente cuando las consecuencias de tales conductas puedan trasladarse a terceros. El sistema de incentivos debe abarcar tanto a depositantes, gerencia, accionistas, como así al ente regulador y el sistema judicial.

Sin prestamista de última instancia, o con fondos ilimitados para dar asistencia, los requisitos mínimos de liquidez y capital realzan su importancia. Pero el riesgo sistémico es un problema que va mucho más allá de la existencia (o no) de un prestamista de última instancia. En general, la solidez estructural del sistema financiero es quizás el elemento más importante para que la quiebra de una entidad (por grande que sea) o un shock macroeconómico (por imprevisto que sea) no se convierta en un problema sistémico.

RECONOCIMIENTO

Los autores agradecen la colaboración de Víctor Manuel Poma en cuestiones relacionadas al vocabulario legal y técnico en el área vinculada al Sector Financiero en versiones preliminares de este documento. Las opiniones expresadas en el documento no reflejan necesariamente la visión del grupo BBVA ni la de sus subsidiarias.

\section{REFERENCIAS}

Acemoglu, D., Ozdaglar, A., \& TahbazSalehi, A. (2015). Systemic risk and stability in financial networks. American Economic Review, 105(2), 564-6o8. doi: 10.1257/aer.20130456

Acharya, V. (2015). Financial stability in the broader mandate for central banks: A political economy perspective. Hutchins Center Working Papers. Recuperado de: https:// www.brookings.edu/wp-content/ uploads/2016/o6/14_financial_stability_ central_banks.pdf

Acharya, V., Drechsler, I., \& Schnabl, P. (2014). A pyrrhic victory? Bank bailouts and sovereign credit risk. The Journal of Finance, 69(6), 2689-2739. doi: https:// doi.org/10.1111/jofi.12206

Acharya, V, \& Skeie, D. (2011). A model of liquidity hoarding and term premia in inter-bank markets. Journal of Monetary Economics, 58(5), 436447. doi: https://doi.org/10.1016/j. jmoneco.2011.05.006

Allen, F., \& Gale, D. (2000). Financial contagion. Journal of political economy, 108(1), 1-33. doi: https://doi. org/10.1086/262109

Alter, A., \& Schüler, Y. S. (2012). Credit spread interdependencies of European states and banks during the financial crisis. Journal of Banking \& Finance, 36(12), 3444-3468. doi: https://doi. org/10.1016/j.jbankfin.2012.08.002

Arnaudo, A. (1988). Economía Monetaria. 
Fondo de Cultura Económica 2 ${ }^{\underline{a}}$ Edición. Balassa, B. (1985). Exports, Policy Choices and Economic Growth in Developing Countries after the 1973 Oil Shock. Journal of Development Economics, 18(2), 23-35. doi: https:// doi.org/10.1016/0304-3878(85)90004-5

Brunnermeier, M., \& Oehmke, M. (2013). Bubbles, financial crises, and systemic risk. In Handbook of the Economics of Finance (Vol. 2, pp. 1221-1288). Elsevier. doi: https://doi.org/10.1016/B978-0-44459406-8.00018-4

Bullard, J., Neely, C., \& Wheelock, D. (2009). Systemic risk and the financial crisis: a primer. Federal Reserve Bank of St. Louis Review, 91. Recuperado de: https://files.stlouisfed.org/files/ htdocs/publications/review/og/og/ parti/Bullard.pdf

Cabrera, M., Dwyer, G., \& Nieto, M. (2018). The G-20's Regulatory Agenda and Banks' Risk. Journal of Financial Stability 39, 66-78. doi: https://doi. org/10.1016/j.jfs.2018.09.001

Cabrera, M., Dwyer, G., \& SamartínSaénz, M. (2016). Government finances and bank bailouts: Evidence from European stock markets. Journal of Empirical Finance, 39, 169-179. doi: https://doi.org/10.1016/j. jempfin.2016.06.005

Diamond, D. \& Dybvig, P. (1983). Bank runs, deposit insurance, and liquidity. Journal of political economy, 91(3), 401419. doi: https://doi.org/10.1086/261155

Faria-e-Castro, M., Martinez, J., \& Philippon, T. (2016). Runs versus lemons: information disclosure and fiscal capacity. The Review of Economic Studies, 84(4), 1683-1707. doi: https:// doi.org/10.1093/restud/rdwo6o

Fernández, R. (1996). Prevención del Riesgo Sistémico en Crisis Financieras (No. 112). Universidad del CEMA. Recuperado de: https://ucema. edu.ar/publicaciones/download/ documentos/112.pdf

Fischer, S. (2016). Monetary policy, financial stability, and the zero lower bound. American Economic Review, 106(5), 39-42. doi: 10.1257/aer.p20161005

Freeman, S. (1996). Clearinghouse Banks and Banknote Over-Issue. Journal of Monetary Economics 38 101-15. doi: https://doi.org/10.1016/03043932(96)01269-X
Gardner, R. (1996). Juegos para empresarios y economistas. Antoni Bosh editores, 1. a edición.

Gibbons, R. (1993). Un primer curso de teoría de juegos. Antoni Bosch Editor. Goldstein, I., \& Pauzner, A. (2005). Demand-deposit contracts and the probability of bank runs. The Journal of Finance, $60(3), 1293-1327$. doi: https://doi.org/10.1111/j.15406261.2005.00762.x

Harsanyi, J. (1962). Bargaining in Ignorance of the Opponent's Utility Function. Journal of Conflict Resolution. doi: https://doi. org/10.1177/002200276200600104

Levine, R. (2012). The governance of financial regulation: reform lessons from the recent crisis. International Review of Finance, 12(1), 39-56. doi: https://doi.org/10.1111/j.14682443.2011.01133. $\mathrm{x}$

Morgenstern, O. (1946): "Economics and the Theory of Games"- Kyklos.

Nash, J. (1950). Equilibrium points in $n$-person games. Proceedings of the National Academy of the USA 36(1):4849. Recuperado de: https://web.calpoly. edu/ aamendes/GTweb/Nash.pdf

Osborne, M. \& Rubinstein, A. (1994). A Course in Game Theory. MIT Press, ISBN 0-262-65040-1.

Paltalidis, N., Gounopoulos, D., Kizys, R., \& Koutelidakis, Y. (2015). Transmission channels of systemic risk and contagion in the European financial network. Journal of Banking \& Finance, 61, S36-S52. doi: https://doi. org/10.1016/j.jbankfin.2015.03.021

Rochet, J., \& Tirole, J. (1996). Interbank lending and systemic risk. Journal of Money, credit and Banking, 28(4), 733762. doi: 10.2307/2077918

Rochet, J. and Vives, X. (2004). Coordination failures and the lender of last resort: was bagehot right after all? Journal of the European Economic Association, 2(6):1116.1147. doi: https:// doi.org/10.1162/1542476042813850

Saunders, A., \& Wilson, B. (1996). Contagious bank runs: evidence from the 1929-1933 period. Journal of Financial Intermediation, 5(4), 409-423. doi: https://doi.org/10.1006/ jfin.1996.0022

Shapiro, C., Carl, S., \& Varian, H. (1998). 
Information rules: a strategic guide to the network economy. Harvard Business Press.

Varian, H. (1995). Análisis Macroeconómico. Antoni Bosch Editor

Varian, H. (1995). Microeconomía Intermedia. Antoni Bosch Editor

Vives, X. (2014). Strategic complementarity, fragility, and regulation. Review of Financial Studies, 27(12):3547.3592. doi: https:// doi.org/10.1093/rfs/hhuo64

Von Neumann, J. \& Brown, G. (1953). Solutions of Games by Differential Equations. Contributions to Theory of Games, Vol. I.

Von Neumann, J. \& Morgenstern, O. (1944). Theory of Games and Economic Behavior. 